Journal of Mathematics and Statistics 2 (3): 407-413, 2006

ISSN 1549-3644

(C) 2006 Science Publications

\title{
Stochastic Analysis of a Compound Redundant System Involving Human Failure
}

\author{
${ }^{1}$ Ritu Gupta, ${ }^{2}$ S.K. Mittal and ${ }^{3}$ C. M. Batra \\ ${ }^{1,3}$ Department of Mathematics, Krishna Institute of Engineering and Technology, Ghaziabad, India \\ ${ }^{2}$ Department of Mathematics, M. M. (PG) College, Modinagar, India
}

\begin{abstract}
This study deals with a compound standby redundant system consisting of three subsystems A, B, and C connected in series. The sub-system B consists of one main unit and the other is its standby redundant unit. These units further consist of two sub-units connected in series. The sub units of main unit are connected to sub-units of standby unit through imperfect switching over device. Failure of all sub-systems and repair rate of switching over devices are exponential while repairs of all sub-systems are distributed quite generally. The various reliability parameters have been computed and analyzed by tabular and graphical illustrations.
\end{abstract}

Key words: M.T.T.F., availability, reliability, profit function, standby redundancy

\section{INTRODUCTION}

Reliability is an important concept at the planning, design and operation stages of various complex systems. As long as man has built things, he has wanted to make them as reliable as possible. In practice, we come across with a number of complex systems consisting of one or more parts, failure of any of the parts results in the reduction of efficiency of whole systems or the complete failure of the system and as a result of it, the reliability of the system reduces. The better maintenance of such parts originate better reliability and then only we can achieve the markets demands of reliability, functionality, price and performance of that system. On the other hand it may not be economical to obtain higher order of reliability always through any amount of maintenance. Thus introducing redundant parts and providing maintenance and repair at the time of need may achieve high degree of reliability. In a redundant system, some additional paths are created for the proper functioning of the system. If all the redundant parts start working together at the time of operation, then it is termed as parallel redundancy. A standby redundant system is the one in which one operating unit is followed by spare units called standbys. On the failure of the operating unit, a standby unit is switched on by perfect or imperfect switching device. In the present discussion, the authors have considered a compound system consisting of three sub-systems A, B and C. The sub-system B consists of two units, one is main and the other is its standby. These units further consist of two sub-units viz, (B11, B21) in main unit and (B12, B22) in its standby redundant system. The sub - system B also has two imperfect switching devices S1 and S2. S1 connects B11 and B22 and S2 connects B21 and B12. B12 and
B22 sub-units are same as B11 and B21 respectively. Initially, in sub-system B, the sub-units B11 and B21 are assumed to be in operation. If B11 fails then B12 and B21 may begin to operate through a switching device S2. If B21 fails then B11 and B22 may begin to operate through a switching device S1. The failure in sub-system $\mathrm{C}$ requires waiting time for repair. The system will be in down state due to failure of subsystem A or C or occurrence of any human error. Also, due to failure of two sub-units of one unit and one subunit of other unit of sub-system B, the system suffers complete break down. The Laplace transforms of the time dependent probabilities of the system being in various states have been obtained by employing the supplementary variable technique. Various reliability parameters have been computed and some tabular and graphical illustrations are also given at the end of the paper. The state transition diagram of the system is shown in Fig. a.

\section{Assumptions}

* Initially, the system works perfectly.

* The system consists of three subsystems A, B and $\mathrm{C}$ connected in series.

* The Sub-system B consists of two units, one main unit and other in standby mode.

* The main unit of subsystem B further consists of two sub-units B11 and B21 connected in series. Similarly, standby unit consists of two sub-units B12 \& B22 connected in series.

* The Sub-unit B11 is connected to sub-unit B22 by switch S1 and sub-unit B21 is connected to subunit B12 by switch $\mathrm{S} 2$.

* Imperfect switching device is assumed.

* All the repairs of sub-systems are distributed quite generally while the repair rate of switching over

Corresponding Author: Ritu Gupta, Department of Mathematics, Krishna Institute of Engineering \& Technology, 13 KM Stone, Ghaziabad-Meerut Road, P.O. Muradnagar-201206, Distt. Ghaziabad (U.P.), India, Tel: $0120-2675314,15$ extn. (36) 


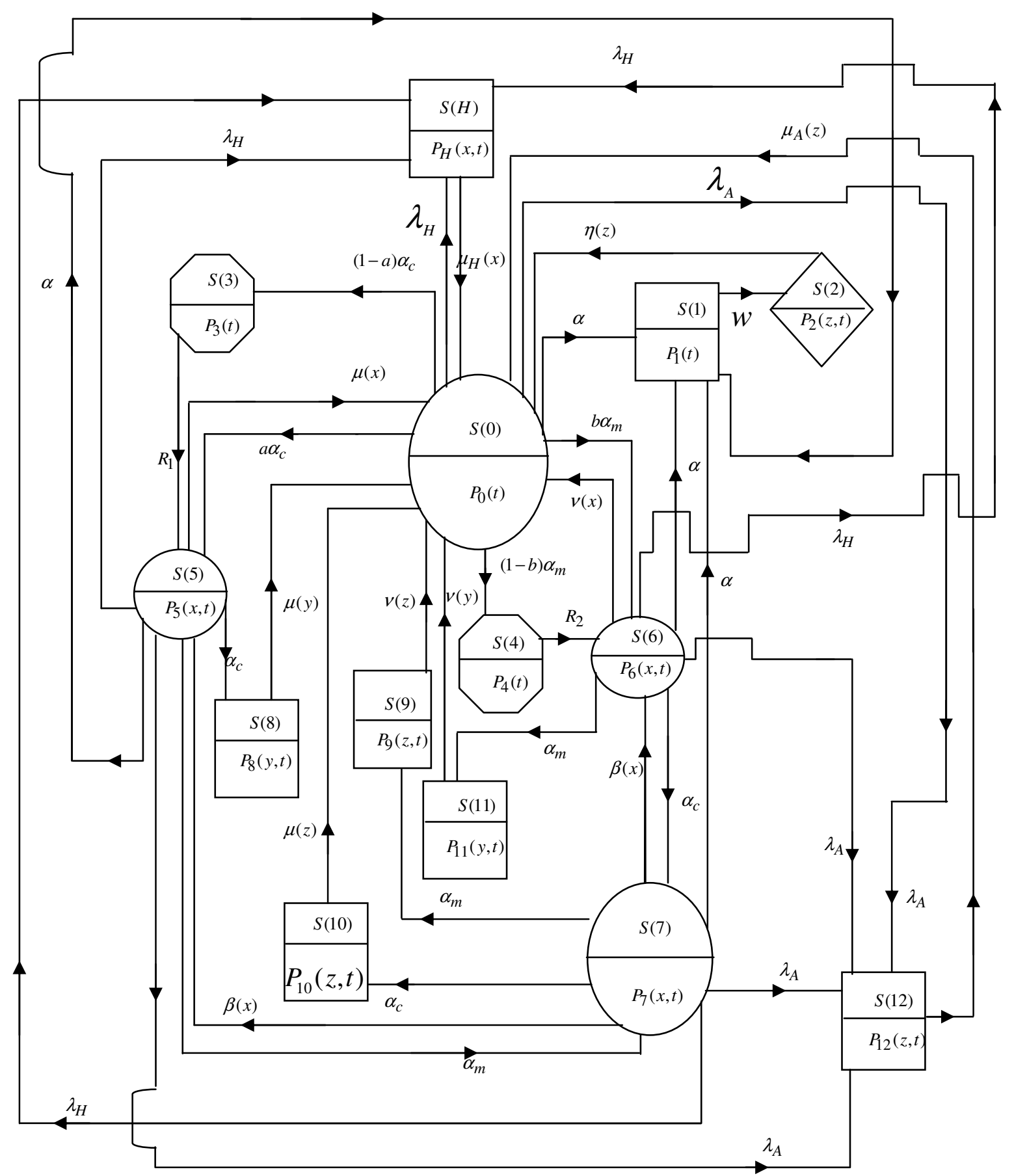

Fig. a: State transition diagram

devices and the failures of sub-systems are exponentially distributed.

* All the units recover their functioning perfectly after repair.

* All the repairs are perfect i.e., the repair facility never does any damage to the units.

* A failed subsystem is repaired at a single service channel.
* At time t=0, B11 and B21 are operating and B12 and $\mathrm{B} 22$ are in standby mode.

* As soon as the operating unit fails, it is replaced by it 's standby unit.

* The system ceases to function due to failure of subsystem A or C or due to occurrence of any human error. 
* The system suffers complete break down due to failure of sub-systems B21, B22 or B11, B12 or any three sub-units of sub-system $B$ at a single state.

* The sub-system $\mathrm{C}$ requires waiting time for repair.

* In state $\mathrm{S}(7)$ of the system no priority is given to any sub-unit i.e., B11, B21 and repair rate of these sub-units is assumed to be identical.

* Standby sub-units in sub-system B are assumed to be perfect as long as they are in standby mode.

\section{Notations}

$\alpha \quad$ : Constant failure rate of subsystem $\mathrm{C}$.

$\eta(z) \quad:$ Repair rate of sub-system $\mathrm{C}$ when the system is in the state $S(2)$.

$w \quad$ : Constant waiting time to repair subsystem C.

$\alpha_{c} \quad:$ Failure rate of sub-unit B21 or B22 of sub-system B.

$\alpha_{m} \quad$ : Failure rate of sub-unit B11 or B12 of sub-system B.

$\lambda_{H}, \mu_{H}(x)$ : Failure and repair rate of the system due to human failure.

$\lambda_{A}, \mu_{A}(z) \quad$ : Failure and repair rate of sub-system A.

$\mu(x) \quad$ : Repair rate of sub-unit B21 when the system is in state $S(5)$.

$\mu(y) \quad$ : Repair rate of sub-unit B21 and B22 when the system is in state $S(8)$.

$\mu(z) \quad$ : Repair rate of sub-unit B11, B21 and B22 when the system is in state $\mathrm{S}(10)$.

$\beta(x) \quad$ : Repair rate of sub-unit B11or $\mathrm{B} 21$ when the system is in state $S(7)$.

$v(x) \quad$ : Repair rate of sub-unit B11 when the system is in state $S(6)$.

$v(y) \quad$ : Repair rate of sub-unit B11 and B12 when the system is in state $S(11)$.

$v(z) \quad$ : Repair rate of sub-unit B11, B21 and B12 when the system is in state $\mathrm{S}(9)$

$x, y, z \quad:$ Elapsed repair time.

$a, b \quad:$ Probability of successful operation of switching over devices $\mathrm{S} 1$ and $\mathrm{S} 2$ respectively.

$R_{1}, R_{2} \quad$ : Constant repair rate of switching over devices $\mathrm{S} 1$ and $\mathrm{S} 2$ respectively.

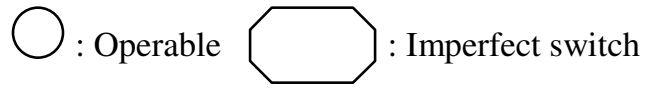

: Failed $>$ Waiting

State probability description

$P_{0}(t) \quad$ : Probability that the system is in operable state $S(0)$ at time ' $t$ '.
$P_{1}(t) \quad$ : Probability that the system is in failed state $\mathrm{S}(1)$ at time $\mathrm{t}$.

$P_{2}(z, t) \Delta$ : Probability that the system is in waiting state $\mathrm{S}(2)$ with elapsed repair time lying in the interval $(z, z+\Delta)$.

$P_{3}(t) \quad$ : Probability that the system is in failed state $\mathrm{S}(3)$ at any time ' $\mathrm{t}$ '

$P_{4}(t) \quad$ : Probability that the system is in failed state $\mathrm{S}(4)$ at any time ' $\mathrm{t}$ '.

$P_{5}(x, t) \Delta:$ Probability that the system is in operable state $\mathrm{S}(5)$ with elapsed repair time lying in the interval $(x, x+\Delta)$.

$P_{6}(x, t) \Delta$ : Probability that the system is in operable state $\mathrm{S}(6)$ with elapsed repair time lying in the interval $(x, x+\Delta)$.

$P_{7}(x, t) \Delta$ : Probability that the system is in operable state $\mathrm{S}(7)$ with elapsed repair time lying in the interval $(x, x+\Delta)$.

$P_{8}(y, t) \Delta:$ Probability that the system is in failed state $\mathrm{S}(8)$ with elapsed repair time lying in the interval $(y, y+\Delta)$.

$P_{9}(z, t) \Delta:$ Probability that the system is in failed state $\mathrm{S}(9)$ with elapsed repair time lying in the interval $(z, z+\Delta)$.

$P_{10}(z, t) \Delta:$ Probability that the system is in failed state $\mathrm{S}(10)$ with elapsed repair time lying in the interval $(z, z+\Delta)$.

$P_{11}(y, t) \Delta:$ Probability that the system is in failed state $\mathrm{S}(11)$ with elapsed repair time lying in the interval $(y, y+\Delta)$.

$P_{12}(z, t) \Delta:$ Probability that the system is in failed state $\mathrm{S}(12)$ with elapsed repair time lying in the interval $(z, z+\Delta)$.

$P_{H}(x, t) \Delta:$ Probability that the system is in failed state $\mathrm{S}(\mathrm{H})$ with elapsed repair time lying in the interval $(x, x+\Delta)$.

Formulation of mathematical model: By elementary probability consideration and continuity arguments, the following difference-differential equations governing the behaviour of the system may be hold good:

$$
\begin{aligned}
& {\left[\frac{d}{d t}+a \alpha_{c}+b \alpha_{m}+(1-b) \alpha_{m}+\lambda_{A}+\alpha+\lambda_{H}+(1-a) \alpha_{c}\right] \cdot P_{0}(t)=\int_{0}^{\infty} \mu(x) \cdot P_{5}(x, t) d x} \\
& +\int_{0}^{\infty} v(x) \cdot P_{6}(x, t) d x+\int_{0}^{\infty} v(y) \cdot P_{11}(y, t) d y+\int_{0}^{\infty} \mu_{A}(z) \cdot P_{12}(z, t) d z+\int_{0}^{\infty} \mu_{H}(z) \cdot P_{H}(x, t) d x \\
& +\int_{0}^{\infty} \eta(z) \cdot P_{2}(z, t) d z+\int_{0}^{\infty} \mu(z) \cdot P_{10}(z, t) d z+\int_{0}^{\infty} v(z) \cdot P_{9}(z, t) d z+\int_{0}^{\infty} \mu(y) \cdot P_{8}(y, t) d y
\end{aligned}
$$




$$
\begin{aligned}
& {\left[\frac{d}{d t}+w\right] \cdot P_{1}(t)=\alpha \cdot P_{0}(t)+\alpha \cdot \int_{0}^{\infty} P_{5}(x, t) d x+} \\
& \alpha \cdot \int_{0}^{\infty} P_{6}(x, t) d x+\alpha \cdot \int_{0}^{\infty} P_{7}(x, t) d x \\
& {\left[\frac{\partial}{\partial z}+\frac{\partial}{\partial t}+\eta(z)\right] \cdot P_{2}(z, t)=0} \\
& {\left[\frac{d}{d t}+R_{1}\right] \cdot P_{3}(t)=(1-a) \alpha_{c} \cdot P_{0}(t)} \\
& {\left[\frac{d}{d t}+R_{2}\right] \cdot P_{4}(t)=(1-b) \alpha_{m} \cdot P_{0}(t)} \\
& {\left[\frac{\partial}{\partial x}+\frac{\partial}{\partial t}+\alpha_{c}+\lambda_{H}+\alpha_{m}+\lambda_{A}+\alpha+\mu(x)\right] \cdot P_{5}(x, t)=0} \\
& {\left[\frac{\partial}{\partial x}+\frac{\partial}{\partial t}+\alpha_{c}+\lambda_{H}+\alpha_{m}+\lambda_{A}+\alpha+v(x)\right] . P_{6}(x, t)=0} \\
& {\left[\frac{\partial}{\partial x}+\frac{\partial}{\partial t}+\alpha_{c}+\lambda_{H}+\alpha_{m}+\lambda_{A}+\alpha+2 \beta(x)\right] \cdot P_{7}(x, t)=0} \\
& {\left[\frac{\partial}{\partial y}+\frac{\partial}{\partial t}+\mu(y)\right] \cdot P_{8}(y, t)=0} \\
& {\left[\frac{\partial}{\partial z}+\frac{\partial}{\partial t}+v(z)\right] \cdot P_{9}(z, t)=0} \\
& {\left[\frac{\partial}{\partial z}+\frac{\partial}{\partial t}+\mu(z)\right] \cdot P_{10}(z, t)=0} \\
& {\left[\frac{\partial}{\partial y}+\frac{\partial}{\partial t}+v(y)\right] \cdot P_{11}(y, t)=0} \\
& {\left[\frac{\partial}{\partial z}+\frac{\partial}{\partial t}+\mu_{A}(z)\right] . P_{12}(z, t)=0} \\
& {\left[\frac{\partial}{\partial x}+\frac{\partial}{\partial t}+\mu_{H}(x)\right] \cdot P_{H}(x, t)=0}
\end{aligned}
$$$$
P_{10}(0, t)=\alpha_{c} \int_{0}^{\infty} P_{7}(x, t) d x
$$$$
P_{11}(0, t)=\alpha_{m} \int_{0}^{\infty} P_{6}(x, t) d x
$$$$
P_{12}(0, t)=\lambda_{A} \int_{0}^{\infty} P_{6}(x, t) d x+\lambda_{A} \cdot P_{0}(t)+
$$$$
\lambda_{A} \int_{0}^{\infty} P_{5}(x, t) d x+\lambda_{A} \int_{0}^{\infty} P_{7}(x, t) d x
$$$$
P_{H}(0, t)=\lambda_{H} \cdot P_{0}(t)+\lambda_{H} \int_{0}^{\infty} P_{5}(x, t) d x+
$$$$
\lambda_{H} \int_{0}^{\infty} P_{6}(x, t) d x+\lambda_{H} \int_{0}^{\infty} P_{7}(x, t) d x
$$

\section{Boundary conditions}

$$
\begin{aligned}
& P_{2}(0, t)=w \cdot P_{1}(t) \\
& P_{5}(0, t)=R_{1} \cdot P_{3}(t)+a \cdot \alpha_{c} \cdot P_{0}(t)+\int_{0}^{\infty} \beta(x) P_{7}(x, t) d x \\
& P_{6}(0, t)=b \cdot \alpha_{m} \cdot P_{0}(t)+R_{2} \cdot P_{4}(t)+\int_{0}^{\infty} \beta(x) P_{7}(x, t) d x \\
& P_{7}(0, t)=\alpha_{c} \int_{0}^{\infty} P_{6}(x, t) d x+\alpha_{m} \int_{0}^{\infty} P_{5}(x, t) d x \\
& P_{8}(0, t)=\alpha_{c} \int_{0}^{\infty} P_{5}(x, t) d x \\
& P_{9}(0, t)=\alpha_{m} \int_{0}^{\infty} P_{7}(x, t) d x
\end{aligned}
$$

Initial conditions: $P_{0}(0)=1$ and other state probabilities are zero.

Solution of the model: Taking Laplace transform of (1) to (24) and on further simplification one may obtain:

$\overline{P_{0}}(s)=\frac{1}{A(s)}$

$\bar{P}_{1}(s)=K_{5}(s) \frac{1}{A(s)}$

$\overline{P_{2}}(s)=K_{6}(s) \cdot \frac{1}{A(s)}$

$\overline{P_{3}}(s)=K_{7}(s) \cdot \frac{1}{A(s)}$

$\overline{P_{4}}(s)=K_{8}(s) \cdot \frac{1}{A(s)}$

$\overline{P_{5}}(s)=K_{12}(s) \cdot \frac{1}{A(s)}$

$\overline{P_{6}}(s)=K_{13}(s) \cdot \frac{1}{A(s)}$

$\overline{P_{7}}(s)=K_{14}(s) \cdot \frac{1}{A(s)}$

$\overline{P_{8}}(s)=K_{15}(s) \cdot \frac{1}{A(s)}$

$\overline{P_{9}}(s)=K_{16}(s) \cdot \frac{1}{A(s)}$

$\overline{P_{10}}(s)=K_{17}(s) \cdot \frac{1}{A(s)}$ 


$$
\begin{aligned}
& \overline{P_{11}}(s)=K_{18}(s) \cdot \frac{1}{A(s)} \\
& \overline{P_{12}}(s)=K_{20}(s) \cdot \frac{1}{A(s)} \\
& \overline{P_{H}}(s)=K_{21}(s) \cdot \frac{1}{A(s)},
\end{aligned}
$$

Operational availability and non-availibility: The Laplace transform of the probabilities that the system is in operable and down state at time ' $t$ ' can be evaluated as follows:

$$
\begin{aligned}
& \bar{P}_{u p}(s)=\frac{1}{A(s)}\left\{1+K_{12}(s)+K_{13}(s)+K_{14}(s)\right\} \\
& \bar{P}_{\text {down }}(s)=\frac{1}{A(s)}\left\{\begin{array}{l}
K_{5}(s)+K_{6}(s)+K_{7}(s)+K_{8}(s)+K_{15}(s)+K_{16}(s)+K_{17}(s) \\
+K_{18}(s)+K_{20}(s)+K_{21}(s)
\end{array}\right\}
\end{aligned}
$$

It is worth noticing that $\bar{P}_{u p}(s)+\bar{P}_{\text {down }}(s)=\frac{1}{S}$.

Where,

$$
\begin{aligned}
& A(s)=\left(s+\alpha_{c}+\alpha_{m}+\lambda_{A}+\lambda_{H}+\alpha\right)- \\
& K_{22}(s)-K_{23}(s)-K_{24}(s)- \\
& K_{25}(s)-K_{26}(s)-K_{27}(s) \\
& -K_{28}(s)-K_{29}(s)-K_{30}(s) \\
& K_{1}(s)=\frac{1}{\left(s+\alpha_{c}+\lambda_{H}+\alpha_{m}+\lambda_{A}+\alpha+\mu(x)\right)} \\
& K_{2}(s)=\frac{1}{\left(s+\alpha_{c}+\lambda_{H}+\alpha_{m}+\lambda_{A}+\alpha+v(x)\right)} \\
& K_{3}(s)=\frac{1}{\left(s+\alpha_{c}+\lambda_{H}+\alpha_{m}+\lambda_{A}+\alpha+2 \beta(x)\right)} \\
& K_{4}(s)=\beta(x) \cdot K_{3}(s) \\
& K_{5}(s)=\frac{\alpha}{s+w}+\alpha \cdot K_{12}(s)+\alpha \cdot K_{13}(s)+\alpha \cdot K_{14}(s) \\
& K_{6}(s)=\frac{w}{(s+\eta(z))} \cdot K_{5}(s) \\
& K_{7}(s)=\frac{(1-a) \alpha_{c}}{\left(s+R_{1}\right)} \\
& K_{8}(s)=\frac{(1-b) \alpha_{m}}{\left(s+R_{2}\right)} \\
& K_{9}(s)=1-\alpha_{c} \cdot K_{4}(s) \cdot K_{2}(s)-\alpha_{m} \cdot K_{4}(s) \cdot K_{1}(s) \\
& K_{10}(s)=K_{2}(s)\left\{\alpha_{c} \cdot b \cdot \alpha_{m}+\alpha_{c} \cdot R_{2} \cdot K_{8}(s)\right\} \\
& +K_{1}(s)\left\{\alpha_{m} \cdot R_{1} \cdot K_{7}(s)+\alpha_{m} \cdot a \cdot \alpha_{c}\right\} \\
& K_{11}(s)=\frac{K_{10}(s)}{K_{9}(s)} \\
& K_{12}(s)=K_{1}(s)\left\{K_{7}(s) \cdot R_{1}+a \cdot \alpha_{c}+K_{11}(s) \cdot K_{4}(s)\right\} \\
& K_{13}(s)=K_{2}(s)\left\{K_{8}(s) \cdot R_{2}+b \cdot \alpha_{m}+K_{11}(s) \cdot K_{4}(s)\right\} \\
& K_{14}(s)=K_{11}(s) \cdot K_{3}(s) \\
&
\end{aligned}
$$

$$
\begin{aligned}
& K_{15}(s)=\frac{\alpha_{c} \cdot K_{1}(s)}{(s+\mu(y))}\left\{K_{7}(s) \cdot R_{1}+a \cdot \alpha_{c}+K_{11}(s) \cdot K_{4}(s)\right\} \\
& K_{16}(s)=\frac{\alpha_{m} \cdot K_{11}(s) \cdot K_{3}(s)}{(s+v(z))} \\
& K_{17}(s)=\frac{\alpha_{c} \cdot K_{11}(s) \cdot K_{3}(s)}{(s+\mu(z))} \\
& K_{18}(s)=\frac{\alpha_{m} \cdot K_{13}(s)}{(s+v(y))} \\
& K_{19}(s)=1+K_{12}(s)+K_{13}(s)+K_{14}(s) \\
& K_{20}(s)=\frac{\lambda_{A} \cdot K_{19}(s)}{\left(s+\mu_{A}(z)\right)} \\
& K_{21}(s)=\frac{\lambda_{H} \cdot K_{19}(s)}{\left(s+\mu_{H}(x)\right)} \\
& K_{22}(s)=\frac{\mu(x)}{\left(s+\alpha_{c}+\lambda_{H}+\alpha_{m}+\lambda_{A}+\alpha+\mu(x)\right)} \\
& \left\{K_{7}(s) \cdot R_{1}+a \cdot \alpha_{c}+K_{11}(s) \cdot K_{4}(s)\right\} \\
& K_{23}(s)=\frac{v(x)}{\left(s+\alpha_{c}+\lambda_{H}+\alpha_{m}+\lambda_{A}+\alpha+v(x)\right)} \\
& \left\{K_{8}(s) \cdot R_{2}+b \cdot \alpha_{m}+K_{11}(s) \cdot K_{4}(s)\right\} \\
& K_{24}(s)=\alpha_{m} \cdot K_{13}(s) \frac{v(y)}{(s+v(y))} \\
& K_{25}(s)=\lambda_{A} \cdot K_{19}(s) \frac{\mu_{A}(z)}{\left(s+\mu_{A}(z)\right)} \\
& K_{26}(s)=\lambda_{H} \cdot K_{19}(s) \frac{\mu_{H}(x)}{\left(s+\mu_{H}(x)\right)} \\
& K_{27}(s)=\frac{w \cdot \alpha \cdot \eta(z)}{(s+w)(s+\eta(z))} \\
& K_{28}(s)=\alpha_{c .} K_{11}(s) \cdot K_{3}(s) \frac{\mu(z)}{(s+\mu(z))} \\
& K_{29}(s)=\alpha_{m} K_{11}(s) \cdot K_{3}(s) \frac{v(z)}{(s+v(z))} \\
& K_{30}(s)=\frac{\alpha_{c} \cdot K_{1}(s) \cdot \mu(y)}{(s+\mu(y))} \\
& \left\{K_{7}(s) \cdot R_{1}+a \cdot \alpha_{c}+K_{11}(s) \cdot K_{4}(s)\right\}
\end{aligned}
$$

Ergodic behaviour: Using Abel's lemma in Laplace transform, viz $\quad \lim _{s \rightarrow 0}(s \cdot \bar{f}(s))=\lim _{t \rightarrow \infty} f(t)=f($ say $)$, provided that the limit on the right hand side exists, the time independent up and down state probabilities are obtained as follows:

$$
\begin{aligned}
& P_{u p}=\frac{1}{A^{\prime}(0)}\left\{K_{12}(0)+K_{13}(0)+K_{14}(0)\right\} \\
& P_{\text {down }}=1-P_{u p}
\end{aligned}
$$

Where 
$A^{\prime}(0)=1-K_{2}^{\prime}(0)-K_{23}^{\prime}(0)-K_{24}^{\prime}(0)-K_{25}^{\prime}(0)$

$-K_{26}^{\prime}(0)-K_{27}^{\prime}(0)-K_{28}^{\prime}(0)-K_{29}^{\prime}(0)-K_{30}^{\prime}(0)$

Reliability: The reliability of the system is given as follows:

$R(t)=e^{-a_{1} \cdot t}+\left(a \cdot \alpha_{c}+b \cdot \alpha_{m}\right) \cdot t \cdot e^{-a_{1} \cdot t}$

$+\left(\alpha_{c} \cdot b \cdot \alpha_{m}+\alpha_{m} \cdot \alpha_{c} \cdot a\right) \cdot \frac{t^{2}}{2} \cdot e^{-a_{1} \cdot t}$

Where $a_{1}=\alpha_{c}+\alpha_{m}+\lambda_{A}+\lambda_{H}+\alpha$

M.T.T.F: The Mean Time To Failure of the system is given by

M.T.T.F. $=\int_{0}^{\infty} R(t) d t=\frac{1}{a_{1}}+\frac{\left(a \cdot \alpha_{c}+b \cdot \alpha_{m}\right)}{a_{1}^{2}}$

$+\frac{\left(a \cdot \alpha_{c} \cdot \alpha_{m}+b \cdot \alpha_{m} \cdot \alpha_{c}\right)}{a_{1}^{3}}$

\section{Profit function}

i. For Non - repairable system, the Profit Function $H(t)$ in the interval $(0, \mathrm{t})$ is given as follows:

$H(t)=C_{1} \cdot \int_{0}^{t} R(t) d t-C_{2} \cdot t-C_{3}$

ii. For repairable system, the profit function $H(t)$ in the interval $(0, t)$ is given by

$H(t)=C_{1} \cdot \int_{0}^{t} P_{u p}(t) d t-C_{2} . t-C_{3}$

where $C_{1}, C_{2}$ and $C_{3}$ are revenue per unit time, service cost per unit time and system establishment cost respectively.

\section{Numerical computation}

1. Availability analysis: Setting the values

$\alpha_{c}=0.001, \alpha_{m}=0.01, \lambda_{A}=0.01, \lambda_{H}=0.001$,

$\alpha=0.001, a=0.95, b=0.97, R_{1}=R_{2}=1$

$\mu(x)=0.9, v(x)=0.8, \beta(x)=1$,

and taking the inverse laplace transform of (39) the availability of the system is obtained as follows:

$$
\begin{aligned}
& P_{u p}(t)=e^{-0.0231 t}+0.00095\left(\begin{array}{l}
0.707 e^{-t}-1.88 e^{-0.923 t} \\
+0.88 e^{-0.023 t}
\end{array}\right)+ \\
& 0.0097\left(0.1734 e^{-t}-1.462 e^{-0.823 t}+1.288 e^{-0.023 t}\right)
\end{aligned}
$$

The values of $P_{u p}(t)$ for different values of $\mathrm{t}$ are calculated from (47) and have been shown in the Table 1.

\begin{tabular}{lc} 
Table 1: & Variation of availability with time \\
\hline Time $(\mathrm{t})$ & Availability $\left[\mathrm{P}_{\mathrm{up}}(\mathrm{t})\right]$ \\
\hline 0 & 0.999716 \\
1 & 0.984218 \\
2 & 0.965074 \\
3 & 0.944572 \\
4 & 0.923734 \\
5 & 0.903014 \\
6 & 0.882607 \\
7 & 0.862594 \\
8 & 0.843005 \\
9 & 0.823848 \\
10 & 0.805121 \\
\hline
\end{tabular}

Table 2: Variation of reliability with time

\begin{tabular}{lc}
\hline Time $(\mathrm{t})$ & Reliability[R(t)] \\
\hline 0 & 1 \\
1 & 0.98768 \\
2 & 0.975421 \\
3 & 0.963227 \\
4 & 0.951101 \\
5 & 0.939045 \\
6 & 0.927063 \\
7 & 0.915156 \\
8 & 0.903328 \\
9 & 0.89158 \\
10 & 0.879914 \\
\hline
\end{tabular}

Table 3: Variation of profit function(non repairable system) with time

\begin{tabular}{lccc}
\hline Time $(\mathrm{t})$ & \multicolumn{2}{c}{ Profit Function[H(t)] $($ Non Repairable System) } \\
& $-\mathrm{C}_{2}=10.0$ & $\mathrm{C}_{2}=12.5$ & $\mathrm{C}_{2}=15.0$ \\
\hline 1 & 48.363598 & 45.863598 & 43.363598 \\
2 & 111.065918 & 106.065918 & 101.065918 \\
3 & 173.042877 & 165.542877 & 158.042877 \\
4 & 232.48999 & 222.48999 & 212.48999 \\
5 & 289.529999 & 277.029999 & 264.529999 \\
6 & 344.540771 & 329.540771 & 314.540771 \\
7 & 397.808899 & 380.308899 & 362.808899 \\
8 & 449.51181 & 429.51181 & 409.51181 \\
9 & 499.756104 & 477.256104 & 454.756104 \\
10 & 548.609375 & 523.609375 & 498.609375 \\
\hline
\end{tabular}

Table 4: Variation of profit function(repairable system) with time

Time (t) Profit Function[H(t)] (Repairable System)

\begin{tabular}{lrrr} 
& $\mathrm{C}_{2}=10.0$ & $\mathrm{C}_{2}=15.0$ & \multicolumn{1}{c}{$\mathrm{C}_{2}=20.0$} \\
\hline 1 & 34.621096 & 29.621096 & 24.621096 \\
2 & 73.362469 & 63.362469 & 53.362469 \\
3 & 111.106537 & 96.106537 & 81.106537 \\
4 & 147.814384 & 127.814384 & 107.814384 \\
5 & 183.482086 & 158.482086 & 133.482086 \\
6 & 218.121094 & 188.121094 & 158.121094 \\
7 & 251.749405 & 216.749405 & 181.749405 \\
8 & 284.387589 & 244.387589 & 204.387589 \\
9 & 316.057129 & 271.057129 & 226.057129 \\
10 & 346.779572 & 296.779572 & 246.779572 \\
\hline
\end{tabular}

2. Reliability analysis: Setting the values $\alpha_{c}=0.001, \alpha_{m}=0.01, \lambda_{A}=0.01, \lambda_{H}=0.001, \alpha=0.001$, $a=0.95, b=0.97$ and taking different values of $\mathrm{t}$ in (43) one may obtain the reliability of the system as shown in the Table 2 . 
Table 5: Variation of M. T. T. F. with failure rate of B11(or B12) unit M.T.T.F.

\begin{tabular}{llll}
$\underline{\alpha_{m}}$ & $\underline{\lambda_{A}=0.01}$ & $\underline{\lambda_{A}=0.02}$ & $\underline{\lambda_{A}=0.03}$ \\
\hline 0.01 & 65.188622 & 40.616913 & 29.257174 \\
0.02 & 50.058437 & 34.744743 & 26.370426 \\
0.03 & 40.232311 & 29.952579 & 23.674549 \\
0.04 & 33.534729 & 26.195274 & 21.355236 \\
0.05 & 28.716002 & 23.224821 & 19.394199 \\
0.06 & 25.094406 & 20.835817 & 17.734848 \\
0.07 & 22.277441 & 18.880236 & 16.321507 \\
0.08 & 20.025625 & 17.253395 & 15.107628 \\
0.09 & 18.185286 & 15.880587 & 14.05611 \\
0.1 & 16.653547 & 14.707581 & 13.137749 \\
\hline
\end{tabular}

Table 6: Variation of M. T. T. F. with human failure rate M.T.T.F.

\begin{tabular}{llll}
$\underline{\lambda_{H}}$ & $\underline{\alpha=0.001}$ & $\underline{\alpha=0.005}$ & $\underline{\alpha=0.010}$ \\
\hline 0.001 & 65.188622 & 52.621555 & 42.236332 \\
0.002 & 61.545139 & 50.173107 & 40.616913 \\
0.003 & 58.268799 & 47.933498 & 39.113071 \\
0.004 & 55.308372 & 45.877781 & 37.71312 \\
0.005 & 52.621552 & 43.98476 & 36.406895 \\
0.006 & 50.173103 & 42.236324 & 35.185478 \\
0.007 & 47.933491 & 40.616913 & 34.041042 \\
0.008 & 45.877781 & 39.113071 & 32.966671 \\
0.009 & 43.98476 & 37.71312 & 31.956251 \\
0.01 & 42.236324 & 36.406895 & 31.004337 \\
\hline
\end{tabular}

Table 7: Variation of M. T. T. F. with human failure rate M.T.T.F.

\begin{tabular}{llll}
$\underline{\lambda_{H}}$ & $\underline{\lambda_{A}=0.01}$ & $\underline{\lambda_{A}=0.02}$ & $\underline{\lambda_{A}=0.03}$ \\
\hline 0.001 & 65.188622 & 40.616913 & 29.257174 \\
0.002 & 61.545139 & 39.113071 & 28.453701 \\
0.003 & 58.268799 & 37.71312 & 27.692183 \\
0.004 & 55.308372 & 36.406895 & 26.969467 \\
0.005 & 52.621552 & 35.185478 & 26.282713 \\
0.006 & 50.173103 & 34.041042 & 25.629339 \\
0.007 & 47.933491 & 32.966671 & 25.007013 \\
0.008 & 45.877781 & 31.956251 & 24.413599 \\
0.009 & 43.98476 & 31.004337 & 23.847164 \\
0.010 & 42.236324 & 30.106091 & 23.305927 \\
\hline
\end{tabular}

3. Profit function

Non-repairable system: Setting the values $\alpha_{c}=0.001$, $\alpha_{m}=0.01, \lambda_{A}=0.01 \begin{aligned} & \lambda_{H}=0.001, \alpha=0.001, \\ & a=0.95, b=0.97\end{aligned}$, $C_{1}=50.0, C_{3}=5.0$, using (43), taking different values of $C_{2}$ and $\mathrm{t}$ in (45), one may obtain the variation of profit function of the system as shown in the Table 3.

Repairable system: Using (47), taking $C_{1}=50.0, C_{3}=5.0$, different values of $C_{2}$ and $\mathrm{t}$ in (46), one may obtain the variation of profit function of the system as shown in the Table 4 .

4. M.T.T.F analysis: Setting the values $\alpha_{c}=0.001, \lambda_{H}=0.001, \alpha=0.001, a=0.95, b=0.97$ and taking different values of $\lambda_{A}$ in (44), one may obtain the variations of M.T.T.F. of the system against the failure rate of unit $\mathrm{B} 11$ (or B12), $\alpha_{m}$ shown in Table 5.

Setting the values $\alpha_{c}=0.001, \alpha_{m}=0.01, \lambda_{A}=0.01, a=0.95, b=0.97$ and taking different values of $\alpha$ in (44), one may obtain the variations of M.T.T.F. of the system against the human failure rate $\lambda_{H}$ shown in Table 6 .

$$
\text { Setting the values }
$$
$\alpha_{c}=0.001, \alpha_{m}=0.01, \alpha=0.001, a=0.95, b=0.97$ and taking different values of $\lambda_{A}$ in (44), one may obtain the variations of M.T.T.F. of the system against the human failure rate $\lambda_{H}$ shown in Table 7 .

\section{RESULTS}

Table 1exhibits that the operational availability of the system decreases with increase in time period.

Table 2 shows that the reliability of the system decreases as the time period increases.

Table 3 and 4 show the variation in profit function with the increase in time period for non - repairable and repairable system respectively. The series of curve in both the figures exhibits that the profit function decreases with the increase in service cost of the system.

Table 5 depicts the variation of M.T.T.F. with the failure rate of subsystem B11 (or B12). The series of curve represents that M.T.T.F. decreases as the failure rate of sub - system A increases.

Table 6 depicts the variation of M.T.T.F. with human failure rate. The series of curve represents that M.T.T.F. decreases as the failure rate of subsystem $\mathrm{C}$ increases.

Table 7 depicts the variation of M.T.T.F. with the human failure rate. The series of curve represents that M.T.T.F. decreases as the failure rate of subsystem A increases.

\section{ACKNOWLEDGEMENT}

Authors are grateful to Rear Admiral Dr. Ajay Sharma (Director General) and Prof. G. S. Sandhu (Director), of Krishna Institute of Engineering \& Technology, Ghaziabad (India) for their constant encouragement, and providing necessary facilities (including financial support) to carry out the present work. The authors do not have any other industrial links.

\section{REFERENCES}

1. Masanori, K., J. Fukata and I. Sawa, 1985. Reliability consideration on a compound redundant system with repair. Microelectronics Reliability, 25: 485-510.

2. Calabro, S.R. Reliability Principles and Practice. McGraw Hill Book Company, Inc. New York.

3. Chung, W.K., 1987. Reliability analysis of a repairable parallel system with standby involving human error and common cause failure. Microelectronics Reliability, 27: 269-274. 